\title{
Influencia de las variables sociodemográficas sobre la ansiedad y el rendimiento académico adolescente: el contexto pluricultural de Ceuta
}

\author{
Influence of Sociodemographic Variables on Anxiety and Academic \\ Achievement in Adolescence: The Pluricultural Context of Ceuta
}

\author{
Federico Pulido Acosta ${ }^{1}$ \\ Francisco Herrera Clavero ${ }^{2}$ \\ Universidad de Granada, Departamento de Psicología Evolutiva y de la Educación, Granada, España
}

\begin{abstract}
Resumen. Este trabajo tiene como objetivo fundamental analizar la ansiedad y el rendimiento académico, sus tipos y niveles en función de edad, género, cultura y estrato socioeconómico, así como contemplar las relaciones entre ambas. Se contó con 811 participantes. El 71.6\% de la muestra eran musulmanes, mientras que el 28.4\% eran cristianos; el $46.1 \%$ hombres y el 53.9\% mujeres. Como instrumentos de evaluación se empleó el STAIC (Spielberger, 2001) y las calificaciones del alumnado. Se reflejan niveles medio-bajos en ansiedad y medios en rendimiento académico. En la ansiedad influyen edad, género, cultura/religión y estatus socio-económico-cultural. Sobre el rendimiento actúan la edad, la cultura y el estatus. No se halló relación estadísticamente significativa entre ansiedad y rendimiento académico.
\end{abstract}

Palabras clave. Ansiedad, rendimiento académico, diversidad, contexto pluricultural.

Abstract. The main objective of this paper is to analyze the types and levels of Anxiety and Academic Achievement according to age, gender, culture and socioeconomic and cultural, as well as the relationships between both variables. We focused on 811 participants $71.6 \%$ of whom were Muslims and $28.4 \%$ Christian; $46.1 \%$ male and $53.9 \%$ female. The techniques used in this survey were the STAIC (Spielberger, 2001) as well as the students' grades. The results reflect medium-low levels in Anxiety and medium levels in Achievement. Anxiety is influenced by age, gender, culture and status. Academic Achievement is influenced by age, culture and socioeconomic and cultural. There is no statistically significant relationship between Anxiety and Achievement, except in Mathematics, increasing as the Anxiety scores are lower.

Keywords. Anxiety, academic achievement, diversity, multicultural context.

${ }^{1}$ Federico Pulido Acosta. Universidad de Granada, España. Dirección Postal: Parques de Ceuta, 2a fase, portal 3, $8^{\circ} \mathrm{C}, \mathrm{CP}: 51002$, Ceuta, España. E-mail: feanor_fede@hotmail.com

${ }^{2}$ Francisco Herrera Clavero. Universidad de Granada, España. E-mail: fherrera@ugr.es

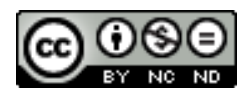

Esta obra está bajo una licencia de Creative Commons Reconocimiento-NoComercial-SinObraDerivada 4.0 Internacional. 


\section{Introducción}

A lo largo de la vida, resulta fundamental saber reaccionar de manera adecuada ante las emociones y saber gestionarlas. A pesar de que, tradicionalmente se considera el aspecto emocional como un elemento que podía llegar a hacer a las personas más débiles, en la actualidad se convierte en un arma muy importante con la que afrontar de manera exitosa la vida. El manejo de la ansiedad cobra relevancia en este sentido.

Dentro del campo emocional Gómez-Ortiz, Casas y Ortega-Ruiz (2016) consideran el estado de ansiedad como la respuesta fisiológica que anticipa la amenaza para el bienestar del sujeto. Puede ser de una intensidad variable y constituir un deterioro en las actividades cotidianas, dando paso al inicio de un trastorno emocional y reduciendo el rendimiento del sujeto en múltiples ámbitos. Esto hace que se pueda considerar la teoría de la ansiedad estado-rasgo, que plantea una relación clara entre ambos conceptos. La primera se refiere a elementos emocionales de carácter más puntual, una condición emocional transitoria; mientras que, la otra, se aproximaría más a la consideración de estado emocional, por su carácter estable.

Es importante considerar las consecuencias desfavorables que pueden ir asociadas a los estados emocionales de carácter negativo. La ansiedad, por ejemplo, puede dificultar el adecuado desarrollo del autoconcepto, llegando a suponer una falta de aceptación por parte de los demás, dificultades en el proceso de socialización y malestar (Gómez-Ortiz et al., 2016). Estos estados emocionales también pueden ser responsables de problemas académicos (Ranoyen, Jozefiak, Wallander, Lydersen, \& Indredavik, 2014).

En referencia a la ansiedad, y teniendo en cuenta las variables sociodemográficas que se consideraron en el trabajo realizado, se puede comprobar un descenso progresivo en el diagnóstico de problemas de ansiedad con la edad del sujeto, considerándola como elemento de riesgo en dicho diagnóstico (Gómez-Ortiz et al., 2016). De esta forma, se remarca el descenso de sus niveles a medida que el sujeto va aumentando su edad, por lo que se llega a aconsejar un análisis por separado para sujetos de diferentes edades.

Se considerará en este trabajo la clara relación de la ansiedad con el género, considerada otra de las variables sociodemográficas con gran relevancia dentro de este campo, pues se encuentran diferencias significativas. Así, las mujeres presentan niveles superiores de ansiedad (Moreno-Rosset, Arnal-Remón, Antequera-Jurado, \& Ramírez-Uclés, 2016; Pulido \& Herrera, 2016). Esta realidad es tan extendida, varios autores (Matesanz, 2006; Gómez-Ortiz et al., 2016) son partidarios de la evaluación así como la realización del análisis estadístico por separado para ambos géneros.

Ante esto, se debe mirar hacia la diversidad de roles y la variación de los estilos de crianza entre hombres y mujeres. Otra de las variables que se tuvieron en cuenta en este estudio, por ser especialmente significativa en el contexto en el que se desarrolló, es la cultura de pertenencia. Esto porque existe influencia de la cultura sobre el desarrollo emocional y, por tanto, sobre la manifestación de diferentes estados emocionales como la ansiedad. Esta realidad cobra más sentido teniendo en cuenta que las aulas son reflejo de la amplia diversidad cultural y social que caracteriza a la sociedad actual (Balongo \& Mérida, 2016). 
Al mismo tiempo, la religión y las normas aprendidas en el seno familiar hacen que se asuman, sin cuestionarse otro punto de vista diferente, los patrones ofrecidos dentro del seno familiar (Soriano \& González, 2013). Por todo ello, se vuelve a remarcar la importancia del contexto socio-familiar en el que el sujeto se desarrolla, como predictor de diferencias significativas en las manifestaciones emocionales (Caballero-Dominguez, Suárez-Colorado, \& Bruges-Carbono, 2015).

Todo lo comentado para la cultura se relaciona de forma directa con el estatus socioeconómico y cultural. En este sentido, se debe considerar que la población musulmana ocupa, de manera predominante, los estratos socioeconómicos y culturales más bajos, destacando por sus graves dificultades de enculturación y por el uso de una lengua diferente al castellano (dariya, variedad del árabe), así como una presencia muy marcada de su religión. Por esta razón, hay una importante vinculación entre la cultura y el estatus. De esta manera, son diferentes los estudios en los que se ha encontrado un nivel superior de ansiedad entre los sujetos pertenecientes a estratos socioeconómicos más bajos (Roth et al., 2014).

Con respecto a los diferentes instrumentos de evaluación de la ansiedad, se dividen en tres apartados. En primer lugar, aparecerían las entrevistas realizadas tanto a padres como a profesores y alumnos. En segundo lugar, se encuentran los autorregistros. Finalmente, aparecen los que constituyen el procedimiento más utilizado para evaluar la ansiedad, por su corto periodo de aplicación, el amplio abanico de elementos emocionales que se pueden contemplar y su sensibilidad para detectar la eficacia terapéutica: los cuestionarios, inventarios o escalas (Martínez-Monteagudo, Inglés, \& García-Fernández, 2013). Dentro de ellos, el más empleado es el Inventario de Ansiedad Estado-Rasgo para Niños (STAIC, State-Trait Anxiety Inventory for Children, Spielberger, 2001).

En otro punto, se introduce la concepción de los investigadores sobre el concepto de rendimiento académico, que se refiere al nivel de conocimientos y destrezas escolares exhibidos por los estudiantes. De la misma forma que se ha realizado para la variable ansiedad, se ofrece la perspectiva del rendimiento en función de las variables sociodemográficas que se considerarán. Desde esta perspectiva, y sobre el rendimiento académico, Pulido y Herrera (2016) establecen diferencias en función del género, pues actúa como predictor del rendimiento.

En este trabajo fueron las mujeres las que presentan mejores resultados que los hombres. Sin embargo, la cultura es un factor mucho más determinante en las calificaciones académicas. De nuevo, Pulido y Herrera (2016) demostraron diferencias en los niveles -no solo en el rendimiento académico general, sino también en cada una de las materias- en dos muestras de culturas diferentes.

Otro de los elementos considerados como determinantes (Pulido \& Herrera, 2016) es el estatus, que guarda una estrecha relación con el grupo cultural de pertenencia, y en donde se encuentran, también, diferencias estadísticamente significativas, aumentando las calificaciones con el estatus socioeconómico. Por contra, dentro de las variables consideradas en el trabajo, la edad es la única en la que no se encontraron diferencias significativas (Pulido \& Herrera, 2016). 
En este caso, se van a emplear las calificaciones escolares como indicativo del rendimiento académico. Por esto, se ha de considerar a Gómez-Castro (1986, p. 269), ya que "al comparar los resultados de las calificaciones objetivas a través de una prueba estandarizada y las que otorgan los profesores, indica cierta asociación entre ellas". Todas las correlaciones obtenidas entre las distintas subpruebas y las calificaciones son estadísticamente significativas $(p<.01)$.

Por otro lado, se contempla también una relación entre ansiedad y rendimiento académico, dado que este puede llegar a dificultar el desarrollo educativo, influyendo, de forma negativa en el mismo (Contreras, Espinosa, Esguerra, Haikal, \& Rodríguez, 2005), especialmente cuando aparece en niveles altos. Esta realidad permite que se pueda considerar la ansiedad moderada como elemento con una función útil, si se tiene en cuenta que genera un estado de alerta que facilita el rendimiento académico (Victor \& Ropper, 2002) facilitando el aprendizaje mecánico.

La ansiedad moderada facilitaría el aprendizaje, mientras que un nivel alto pudiera inhibirlo notablemente, especialmente cuando se trata de aprendizajes más complejos, planteando dificultades en los procesos motivacionales y cognitivos que influyen sobre las habilidades y destrezas necesarias en el proceso. En este sentido, Contreras et al. (2005) indican otro aspecto que resulta relevante, contemplando el papel moderado de la ansiedad estado y del papel relativamente consistente de la ansiedad rasgo en el rendimiento académico.

Considerando todo lo mencionado, se presenta el estudio llevado a cabo con una muestra de alumnos escolarizados en Instituto de Enseñanza Secundaria en la ciudad de Ceuta, donde conviven principalmente personas de cultura cristiana y musulmana. Como característica específica, la población árabe musulmana presenta una procedencia marroquí con un altísimo nivel de analfabetismo y una elevada natalidad, así como un estatus socioeconómico y cultural bajo, serios problemas de enculturación y bilingüismo (integración por la comunicación) y con una presencia muy marcada de su religión, el Islam, en sus vidas (Herrera, 2000).

Los objetivos del trabajo han sido analizar los tipos y niveles de ansiedad y rendimiento académico, en función de la edad, género, cultura y estrato socioeconómico, así como contemplar las relaciones que se pudieran dar entre ambas variables, para una población adolescente multicultural. De esta manera, se pretende determinar si la ansiedad, considerando el instrumento que se empleará, aparece como elemento a tener en cuenta en la búsqueda de la mejora del rendimiento académico.

\section{Método}

\section{Participantes}

Para llevar a cabo esta investigación, se seleccionó una muestra integrada por 811 participantes que reflejan las características del contexto pluricultural de Ceuta. Se reparten entre seis centros en los que se imparte formación perteneciente a la Etapa Secundaria. Las características sociodemográficas de la muestra tomada aparecen resumidas en la tabla 1. 
Tabla 1

\begin{tabular}{|c|c|c|c|c|}
\hline \multirow[t]{2}{*}{ Variables sociodemográficas } & \multicolumn{4}{|c|}{ Media descriptiva } \\
\hline & $N=811$ & $M$ & $D T$ & Rango \\
\hline Edad & & 15.39 & 4.46 & $12-47$ \\
\hline \multirow{3}{*}{ Cultura/Religión } & & & $f$ & $\%$ \\
\hline & Cristianos & & 221 & 28.4 \\
\hline & Musulmanes & & 556 & 71.6 \\
\hline \multirow[t]{2}{*}{ Género } & Hombres & & 363 & 46.1 \\
\hline & Mujeres & & 424 & 53.9 \\
\hline \multirow[t]{4}{*}{ Estatus } & Bajo & & 25 & 3.2 \\
\hline & Medio-Bajo & & 173 & 22.3 \\
\hline & Medio & & 521 & 67.2 \\
\hline & Alto & & 56 & 7.2 \\
\hline
\end{tabular}

\section{Procedimiento}

La selección de la muestra se hizo mediante un método aleatorio de sujetos disponibles. Después de solicitar permiso por escrito, tanto a la Administración Educativa, como a la Dirección de los centros y, por supuesto, a los padres de cada alumno menor de edad, se procedió a la recogida de la información, contestando aquellos que lo desearon. Cada uno de los participantes contestó al cuestionario para la evaluación de la ansiedad.

Del mismo modo, los participantes rellenaron una hoja en la que, además de completar sus datos sociodemográficos, reportaron las calificaciones para las materias cursadas. El tiempo total empleado en la contestación fue de 45 minutos. Los participantes se seleccionaron por el método de muestreo incidental o casual. El error muestral fue del 3\%. Finalmente, es necesario indicar que, en el presente trabajo, se cumplió con los estándares éticos que requieren este tipo de investigaciones.

\section{Instrumentos}

Como instrumento para evaluar el miedo se empleó el Inventario de Ansiedad EstadoRasgo para Niños (STAIC, Spielberger, 2001). El STAIC está en dos partes diferenciadas: La Ansiedad Estado (AE), que evalúa cómo se siente la persona en un momento o situación determinados. La Ansiedad Rasgo (AR) evalúa cómo se siente habitualmente. Cada parte del cuestionario queda formada por 20 ítems, con cuatro posibilidades de respuesta cada uno (0: nada, 1: poco, 2: bastante y 3: mucho). Su consistencia interna fue de $\alpha=.934$. Esto también queda demostrado por la prueba de dos mitades de Spearman-Brown, cuyo coeficiente fue de .781 para el cuestionario, incluyendo todos los ítems. 
En el apartado relacionado con la varianza factorial se emplearon diferentes Análisis Factoriales Exploratorios (AFE). En este sentido, los factores obtenidos fueron seis. El primero de los factores se relaciona con los Estados emocionales positivos $(31.53 \%$ de la varianza explicada, perteneciente a la AE); el segundo, queda vinculado a Preocupaciones y dificultades $(9.487 \%, \mathrm{AR})$. El tercer factor se vinculó a los Estados emocionales negativos (4.35\%, AE); el cuarto a los Rasgos emocionales negativos $(3.98 \%$, AR) y el quinto a las Manifestaciones de ansiedad (3.31\%, AE). El último factor conforma la categoría Miedo $(2.99 \%, \mathrm{AE})$. Entre todos los factores (2 corresponden a la AR y 4 a la AE) se consigue una varianza total explicada del 55.676\%.

Se emplearon las calificaciones, como medida del Rendimiento Académico, siguiendo el estudio de Gómez-Castro (1986). Una vez construida la base de datos, se comenzó por el análisis descriptivo, del que se utilizan los porcentajes alcanzados de manera general y en función de las variables sociodemográficas, a través de tablas de contingencia. También, se referencian algunas representaciones gráficas. Para comprobar el nivel de significación de los datos se ha utilizado la prueba de Chi cuadrado de Pearson, a nivel de significación de $\alpha=.05$ junto con ANOVAS de un factor con pruebas post hoc. Finalmente, se muestran los resultados de la regresión múltiple paso a paso o stepwise realizada.

El análisis completo fue posible gracias al programa informático Statistical Package for Social Sciences (SPSS, 2012) y permitió la comprobación del comportamiento de la muestra desde la perspectiva las variables sociodemográficas, así como la interacción entre las variables de estudio. Se comenzó por la comprobación de las características de la muestra, contemplando su distribución en función de las diferentes variables sociodemográficas. Se continuó con el análisis de las propiedades psicométricas de los cuestionarios empleados. Para la presentación de los resultados, se comenzó por el análisis descriptivo, que permite la comprobación de los niveles y tipos generales para cada una de las diferentes variables. A continuación se procedió al análisis inferencial, empleando el análisis regresional (regresiones múltiples paso a paso).

Se consideraron como variables criterio cada una de las variables de estudio (ansiedad y rendimiento académico). Las variables sociodemográficas se emplearon como posibles predictoras. A continuación, se consideraron como variables criterio cada una de las variables de estudio (ansiedad y rendimiento académico). Para determinar la función de regresión, se consideró el último paso y, dentro de él, en el orden de entrada de las variables en la función de regresión, el valor de cada variable, su significación, el índice de regresión $(R)$, el coeficiente de determinación múltiple $\left(R^{2}\right)$ y la varianza explicada de la variable más importante (la que más porcentaje $\beta$ explica).

Para finalizar el análisis inferencial, se concluyó con la prueba t de Student de diferencias de medias para muestras independientes para aquellas variables dicotómicas, mientras que para el resto se empleó el análisis de la varianza (ANOVA de un factor). Al mismo tiempo, se utilizó la prueba de Chi cuadrado de Pearson, aprovechando los resultados obtenidos dentro de las tablas de contingencia que se hicieron para la estadística descriptiva. Para todas las pruebas realizadas se empleó un intervalo de confianza del 95\% (IC 95\%) y un nivel de significación de $p<.05$. Además, en todas las variables con más de dos 
alternativas de respuesta se realizaron diferentes pruebas post hoc (DMS, Bonferroni, Turkey \& Waller-Duncan).

\section{Resultados}

Análisis descriptivo general ansiedad

El comportamiento de la muestra de alumnos de secundaria, en relación con la variable ansiedad, se mueve en puntuaciones bajas y muy bajas. Esto se entiende considerando que la opción de poca ansiedad $(46.7 \%)$ es la más frecuente. La siguiente fue la que refleja muy poca ansiedad (40.9\%) de los encuestados. Los porcentajes de bastante ansiedad (10.7\%) y, sobre todo, los que evidencian niveles más altos (1.5\%), son menores.

\section{Análisis comparativo por edad en ansiedad}

El análisis comparativo por edad indica que el grupo en el que se registran los mayores niveles de ansiedad es precisamente en el de los alumnos de 17-18 años. El siguiente grupo, por nivel, es el de 40 o más, seguido del grupo de 25 a 39 y del de 18 a 25 años. Después, aparece el grupo de 16-17, seguido del de 14-15 años. Seguidamente, aparece el alumnado de entre 15 y 16 y el de 13-14.

El que menos ansiedad evidencia es el alumnado de menor edad. Todo ello queda reflejado por el Análisis de la Varianza (ANOVA de un factor), con la variable edad como variable independiente. Tanto en ansiedad total $(p<.001)$ como en los factores relacionados con esta variable (estados emocionales positivos $p<.001$; preocupaciones y dificultades $p<.001$; estados emocionales negativos $p<.001$; rasgos emocionales negativos $p<.001$; manifestaciones de ansiedad $p<.001 ; \mathrm{y}$ miedo $p<.001)$ se encontraron diferencias estadísticamente significativas y se observó un incremento desde los menores (12-13) hasta los que tienen 17-18 años (periodo que coincide con la adolescencia del sujeto). A continuación, se describe un descenso, volviendo a aumentar los niveles desde este punto hacia los sujetos de más edad.

\section{Análisis comparativo por género en ansiedad}

Considerando la variable ansiedad en función del género, se puede ver que las mujeres tienen un nivel más alto que los hombres. Esta realidad se repite también en cada uno de los factores que integran esta variable, donde las mujeres reflejan medias más altas que los hombres. Una vez hecha esta primera aproximación, se empleó la prueba t de Student de diferencias de medias para muestras independientes.

Considerando el análisis de esta prueba, con la variable género como factor y el resto de las variables mencionadas como dependientes, las diferencias vuelven a ser estadísticamente significativas en ansiedad total $(p<.001)$, en los estados emocionales positivos $(p<.001)$, preocupaciones y dificultades $(p<.001)$, los estados emocionales negativos $(p<.001)$, los rasgos emocionales negativos $(p<.001)$, manifestaciones de ansiedad $(p<.001)$ y miedo $(p<.001)$. En todos los casos, los hombres presentan puntuaciones más bajas.

\section{Análisis comparativo por cultura en ansiedad}

Teniendo en cuenta la cultura/religión, los resultados reflejan que el alumnado perteneciente a la cultura/religión musulmana manifiesta niveles más bajos de ansiedad en 
comparación con los participantes pertenecientes a la religión cristiana. Considerando la prueba t de Student, se pueden afirmar las diferencias estadísticamente significativas en las variables ansiedad total $(p<.05)$ y los factores estados emocionales positivos $(p<.05)$, preocupaciones y dificultades $(p<.05)$ y condiciones de vida negativas $(p<.01)$. Estas se unen a aquellas en las que las diferencias no fueron estadísticamente significativas, como es el caso de los factores estados emocionales negativos, rasgos emocionales negativos, manifestaciones de ansiedad y miedo.

\section{Análisis comparativo por estatus en ansiedad}

Referente al estatus socioeconómico y cultural, se observa una distribución organizada, ya que, por media, los niveles van subiendo a medida que se desciende en el estatus. Los niveles más altos aparecen en el estatus bajo, seguido por el medio-bajo. Después, aparecería el estatus medio. Solo el estatus alto presenta niveles inferiores de ansiedad. De esta forma, las diferencias vuelven a ser estadísticamente significativas en ansiedad total $(p<.01)$ y en los factores relacionados con esta variable: estados emocionales positivos $(p<.05)$, rasgos emocionales negativos $(p<.001)$, estados emocionales negativos $(p<.05)$ y miedo $(p=<.05)$. Estas se unen a aquellas en las que las diferencias no fueron estadísticamente significativas, como es el caso de los factores preocupaciones y dificultades y manifestaciones de ansiedad. Los resultados más relevantes de la prueba ANOVA para la variable ansiedad quedan recogidos en la tabla 2.

Los resultados del cálculo regresional, que se presentan a través de la tabla 3, muestran que en el alumnado de los centros de Educación Secundaria de Ceuta se observa que la variable ansiedad total se la edad y el género. El resto de variables quedan excluidas. El coeficiente de correlación múltiple $(R=.741)$ señala una intensa relación entre la ansiedad y las variables predictora; en conjunto, explican el $54.9 \%$ de la varianza explicada por el modelo $\left(R^{2}=.549\right)$. De todas ellas, la que tiene una mayor importancia es la edad. Su coeficiente de regresión estandarizado demuestra esta influencia, explicando el 19.9\% de la varianza en el modelo $(\beta=.199)$. La edad y el género actúan en sentido positivo. De esta manera, la ansiedad es superior a medida que se asciende en edad y aumenta en el género femenino (dado que se asoció este con la puntuación 2, mientras que los hombres se asociaron al valor 1). La función de regresión y los coeficientes relevantes aparecen a continuación:

$$
\text { Ansiedad Total }=90.976+2.150(\text { Edad })+5.410(\text { Género })
$$

\section{Análisis descriptivo general rendimiento}

Haciendo referencia al rendimiento, establecido por las calificaciones recibidas por el alumnado (nota media), el resultado más habitual (33.7\%) es el notable (entre 7 y menos de 8.5). La siguiente calificación, por porcentaje, es el bien (27.5\%). A continuación, aparecen las calificaciones de aprobado (con un 17.2\%) y sobresaliente (11.1\%). La calificación con porcentajes más bajos $(6.2 \%)$ es el suspenso (entre 1 y 4.5$)$.

Análisis comparativo por edad en rendimiento

Considerando la variable rendimiento, se inicia la descripción del comportamiento de la muestra de alumnos de Secundaria en función de la variable sociodemográfica edad. El grupo con la media más alta es el de entre 16-17 años, que presenta mayor rendimiento 
Tabla 2

\begin{tabular}{|c|c|c|c|c|c|}
\hline Variable & $N$ & Rangos & $M$ & DT & $F / t$ \\
\hline \multicolumn{6}{|c|}{ Edad } \\
\hline \multirow{9}{*}{$\begin{array}{c}\text { Ansiedad } \\
\text { Total }\end{array}$} & 137 & $12-13$ & 29.8759 & 16.31962 & \multirow{9}{*}{$13.349^{* * *}$} \\
\hline & 154 & $13-14$ & 30.0844 & 15.73928 & \\
\hline & 125 & $14-15$ & 38.1120 & 20.51365 & \\
\hline & 74 & $15-16$ & 37.2297 & 16.79412 & \\
\hline & 125 & $16-17$ & 43.4720 & 19.29131 & \\
\hline & 56 & $17-18$ & 51.1250 & 20.37294 & \\
\hline & 96 & $18-25$ & 42.5938 & 18.12795 & \\
\hline & 17 & $25-39$ & 43.9412 & 22.12033 & \\
\hline & 8 & 40 o más & 45.2500 & 15.98884 & \\
\hline \multicolumn{6}{|c|}{ Género } \\
\hline \multirow{2}{*}{$\begin{array}{c}\text { Ansiedad } \\
\text { Total }\end{array}$} & 363 & Hombres & 32.6088 & 16.68546 & \multirow[b]{2}{*}{$47.997 * * *$} \\
\hline & 423 & Mujeres & 41.8345 & 20.11898 & \\
\hline \multicolumn{6}{|c|}{ Cultura } \\
\hline \multirow{2}{*}{$\begin{array}{c}\text { Ansiedad } \\
\text { Total }\end{array}$} & 221 & Cristiana & 39.7919 & 19.00723 & \multirow[b]{2}{*}{$3.982^{*}$} \\
\hline & 555 & Musulmana & 36.7495 & 19.23164 & \\
\hline \multicolumn{6}{|c|}{ Estatus } \\
\hline \multirow{4}{*}{$\begin{array}{c}\text { Ansiedad } \\
\text { Total }\end{array}$} & 25 & Bajo & 48.2000 & 22.71380 & \multirow{4}{*}{$4.716^{* *}$} \\
\hline & 173 & Medio-Bajo & 39.5723 & 20.21920 & \\
\hline & 520 & Medio & 37.0788 & 18.55107 & \\
\hline & 56 & Alto & 32.3750 & 18.58109 & \\
\hline
\end{tabular}

*** $p<.001 ; * * p<.01 ; * p<.05$

que el grupo de 12-13 años, al que sigue el de entre 13-14. A continuación, se coloca el de 18-25 años y muy próximo, el de 17 a 18. El grupo de entre 15-16 supera al siguiente en su media. Los resultados medios más bajos se dan en el alumnado de 14-15 años y, sobre todo en el de 40 o más.

Una situación similar se describe en el rendimiento en las diferentes materias. En todas ellas, el alumnado de entre 16-17 años presenta las puntuaciones más altas. La única excepción es la materia de Matemáticas, donde el grupo de menor edad (12-13) es el que alcanza calificaciones más altas. El Análisis de la Varianza (ANOVA de un factor), con la variable edad como variable independiente y el resto de las variables como dependientes, muestra diferencias estadísticamente significativas en la Media general $(p<.001)$. Lo mismo ocurre con las materias específicas: Lengua Castellana $(p<.001)$, Matemáticas $(p<.001)$, Sociales $(\phi<.05)$, Inglés $(\phi<.001)$, Naturales $(\phi<.001)$ y Religión $(\phi<.001)$. 
Tabla 3

Modelos predictores para la Ansiedad Total

\begin{tabular}{lcccccc}
\hline Predictores & $R$ & $R^{2}$ & $F$ & $B$ & $\beta$ & $t$ \\
\hline & .741 & .549 & 104.587 & & & \\
Constante General & & & & 90.976 & & $19.153^{*}$ \\
Edad & & & & 2.150 & .199 & $6.913^{*}$ \\
Género & & & & 5.410 & .139 & $4.984^{*}$ \\
\hline
\end{tabular}

$* p<.001$

\section{Análisis comparativo por género en rendimiento}

Si se tiene en cuenta la media del rendimiento en función del género, se puede comprobar una enorme igualdad entre hombres y mujeres en cuanto a las calificaciones. Considerando la prueba $t$ de Student de diferencias de medias para muestras independientes, se descartó la significación la media general y todas las materias, pues no se dieron diferencias entre hombres y mujeres.

\section{Análisis comparativo por cultura en rendimiento}

Si se tiene en cuenta la media de rendimiento, en función de la cultura/religión, se puede decir que las calificaciones son claramente superiores entre los cristianos, realidad que se repite en cada una de las materias. Esta realidad queda confirmada por la prueba t de Student, dado que las diferencias fueron estadísticamente significativas en la media general $(p<.001)$, en Lengua Castellana $(p<.001)$, Matemáticas $(p<.01)$, Sociales $(p<.05)$, Inglés $(p<.001)$, Naturales $(p<.001)$ y Religión $(\phi<.001)$. En todos los casos, son los musulmanes los que obtienen resultados más bajos.

\section{Análisis comparativo por estatus en rendimiento}

Considerando la media del rendimiento, dentro de la última de las variables, se observa que las calificaciones aumentan progresivamente a medida que se asciende en el estatus. De esta manera, en el estatus alto es en el que aparecen niveles, seguido del medio. Las calificaciones descienden bastante para los otros dos grupos (medio-bajo y bajo). El estatus alto es en el que aparecen los mejores resultados también en todas las materias. El Análisis de la Varianza (ANOVA de un factor), demostró diferencias estadísticamente significativas en la media general $(p<.001)$, así como en las áreas específicas: Lengua Castellana $(p<.001)$, Matemáticas $(p<.001)$, Sociales $(p<.001)$, Inglés $(p<.001)$, Naturales $(p<.001)$ y Religión $(\phi<.001)$. Los resultados se resumen en la tabla 4 .

Los resultados de la regresión realizada, que reflejan el paso final del método elegido, ponen de manifiesto que la media del rendimiento académico (media de calificaciones) está bajo la influencia de las variables estatus, la cultura/religión y la edad. De todas ellas, actúan en sentido inverso (inversamente proporcional) la cultura/religión y la edad. Esto indica que, a medida que se asciende en edad, y entre los sujetos pertenecientes a la cultura musulmana es inferior la media del rendimiento. 
Tabla 4

ANOVA de Rendimiento como variable dependiente y variables sociodemográficas como independientes

\begin{tabular}{|c|c|c|c|c|c|}
\hline Variable & $N$ & Rangos & $M$ & $D T$ & $F / t$ \\
\hline \multicolumn{6}{|c|}{ Edad } \\
\hline \multirow{9}{*}{$\begin{array}{c}\text { Media } \\
\text { Rendimiento } \\
\text { Académico }\end{array}$} & 127 & $12-13$ & 6.89948 & 1.644860 & \multirow{9}{*}{$7.384^{* * *}$} \\
\hline & 153 & $13-14$ & 6.65741 & 1.625072 & \\
\hline & 120 & $14-15$ & 6.11806 & 1.417082 & \\
\hline & 72 & $15-16$ & 6.20023 & 1.473349 & \\
\hline & 122 & $16-17$ & 7.34563 & 1.193941 & \\
\hline & 43 & $17-18$ & 6.45736 & .977473 & \\
\hline & 72 & $18-25$ & 6.48762 & 1.616180 & \\
\hline & 12 & $25-39$ & 6.15972 & 1.057953 & \\
\hline & 5 & 40 o más & 5.53333 & 1.340605 & \\
\hline \multicolumn{6}{|c|}{ Género } \\
\hline \multirow{2}{*}{$\begin{array}{c}\text { Media } \\
\text { Rendimiento }\end{array}$} & 338 & Hombres & 6.43013 & 1.539395 & \multirow[b]{2}{*}{.185} \\
\hline & 387 & Mujeres & 6.82845 & 1.476712 & \\
\hline \multicolumn{6}{|c|}{ Cultura } \\
\hline \multirow{2}{*}{$\begin{array}{c}\text { Media } \\
\text { Rendimiento }\end{array}$} & 202 & Cristiana & 7.15000 & 1.424128 & \multirow{2}{*}{$31.291^{* * *}$} \\
\hline & 516 & Musulmana & 6.45945 & 1.511363 & \\
\hline \multicolumn{6}{|c|}{ Estatus } \\
\hline \multirow{4}{*}{$\begin{array}{c}\text { Media } \\
\text { Rendimiento }\end{array}$} & 22 & Bajo & 5.38864 & 2.015373 & \multirow{4}{*}{$17.091 * * *$} \\
\hline & 156 & Medio-Bajo & 6.11047 & 1.594503 & \\
\hline & 489 & Medio & 6.84707 & 1.392799 & \\
\hline & 52 & Alto & 7.08654 & 1.381283 & \\
\hline
\end{tabular}

$* * * p<.001$

El estatus es la variable más influyente, cuyo coeficiente de regresión estandarizado es del 14.2\% $(\beta=.142)$. El resto de variables quedan excluidas, explicando entre todas las predictoras el $37.7 \%$ de la varianza total $\left(R^{2}=.377\right)$. La influencia de estas variables es intensa, tal y como indica el coeficiente de correlación $(R=.614)$. La función descrita sería la siguiente, la cual se acompaña con la tabla 5 , que contiene los coeficientes más relevantes:

$$
\text { Media Rendimiento }=1.588+.348 \text { (Estatus })-.276(\text { Cultura } / \text { Religión })-.042(\text { Edad })
$$

Se finaliza el apartado de los resultados, considerando el rendimiento académico, en función de la variable ansiedad. En este sentido, las diferencias son significativas exclusivamente en el rendimiento en Matemáticas $(p<.05)$, descendiendo las calificaciones a medida que se asciende en los niveles de ansiedad. Sin embargo, para la media de rendimiento académico $(\phi>.05)$, así como para el resto de las materias, las diferencias no fueron estadísticamente significativas. Esta realidad queda reflejada en la tabla 6. 
Tabla 5

Modelos predictores para la Media del Rendimiento

\begin{tabular}{|c|c|c|c|c|c|c|}
\hline Predictores & $R$ & $R^{2}$ & $F$ & $B$ & $\beta$ & $t$ \\
\hline & .614 & .377 & 32.818 & & & \\
\hline Constante General & & & & 1.588 & & $2.994^{* *}$ \\
\hline Estatus & & & & .348 & .142 & $4.204^{* * *}$ \\
\hline Cultura/Religión & & & & -.276 & -.085 & $-2.516^{*}$ \\
\hline Edad & & & & -.042 & -.082 & $-1.998 *$ \\
\hline
\end{tabular}

$* * * p<.001 ; * * p<.01 ; * p<.05$

Tabla 6

ANOVA de Rendimiento como variable dependiente y la Ansiedad como independientes

\begin{tabular}{lccccc}
\hline Variable & $N$ & Ansiedad & $M$ & $D T$ & $t$ \\
\hline & & & & & \\
Media & 347 & Muy Poca Ansiedad & 6.68361 & 1.554648 & \\
Rendimiento & 75 & Poca Ansiedad & 6.65204 & 1.474331 & \multirow{2}{*}{ Bastante Ansiedad } \\
& 11 & Mucha Ansiedad & 5.46100 & 1.632872 & \\
& & & &
\end{tabular}

$* p>.05$

\section{Discusión}

En ansiedad se registran diferencias significativas en función de la edad, tanto en las puntuaciones totales, como en todos y cada uno de los factores que integran esta variable. En este caso, las diferencias indican un ascenso desde los 12 hasta los 18 años. Entrando en la etapa adulta, aparecen diferencias menos pronunciadas. Esto indica claramente que la edad es un factor determinante. En todos los casos, el factor preocupaciones y dificultades (AR) y los estados emocionales positivos (AE), vuelven a repartirse los niveles más altos. Esta realidad entra dentro de lo esperado, si se considera la incidencia de los trastornos de ansiedad y su diagnóstico, en relación con la edad (Cazalla-Luna \& Molero, 2014). Resulta relevante mencionar que el ascenso se produce durante la adolescencia del sujeto, periodo destacado por los importantes cambios que experimenta el sujeto. Esta realidad puede dar una posible explicación a las diferencias descritas dentro de esta variable.

En función del género, se vuelven a encontrar diferencias estadísticamente significativas para los totales, así como todos los factores. Como ya se ha comentado, las personas pertenecientes al el género femenino reflejan puntuaciones más elevadas en todos los casos, lo que demuestra que el género es otra de las variables sociodemográficas que se deben tener en cuenta en cualquier investigación que pretenda informar sobre los estados de ansiedad.

El sentido de estas diferencias coincide con la mayoría de trabajos consultados (GómezGaribello \& Chaux, 2014; Quiceno \& Vinaccia, 2015; Moreno-Rosset et al., 2016). Ante estas diferencias encontradas, se pretende un alejamiento de las concepciones basadas en 
criterios de tipo biológico, en las que se remarca la existencia de diferencias hormonales (debidas al ciclo menstrual propio de las mujeres, niveles de estrógeno y progesterona, disminución en los niveles de serotonina, mujeres con una amígdala más desarrollada que los hombres...) indicadas en Pinto, Dutra, Filgueiras, Juruena y Stingel (2013), para concederle mayor credibilidad a aquellas que tienen en cuenta diferencias en los patrones de socialización y la presencia marcada de determinados roles.

También se deben comentar las diferencias en ansiedad, en función de la cultura/ religión del sujeto. En este sentido, aparecieron diferencias estadísticamente significativas en ansiedad total, estados emocionales positivos y preocupaciones y dificultades. Los pertenecientes a la cultura musulmana reflejan niveles inferiores que los pertenecientes a la cristiana-occidental. Esta realidad también apareció en otros trabajos en los que se destaca este contexto como elemento clave para el desarrollo emocional (Pulido \& Herrera, 2016; Gómez-Ortiz et al., 2016). La religión y las normas aprendidas en el seno familiar tienen un importante efecto sobre los patrones ofrecidos dentro por el sujeto, en lo que a crianza de las personas se refiere, lo que justifica estas diferencias en función de esta variable sociodemográfica.

En función del estatus socio-económico y cultural, en ansiedad se observa un descenso progresivo (y los factores estados emocionales positivos, estados emocionales negativos, rasgos emocionales negativos y miedo) a medida que se sube en el Estatus. De esta manera, son varios los estudios en los que se ha encontrado un nivel superior de ansiedad y otros estados emocionales de carácter negativo entre los sujetos pertenecientes a estratos socioeconómicos más bajos (Kushnir, Gothelf, \& Saheh, 2014; Roth et al., 2014; Pulido \& Herrera, 2016).

Continuando con el apartado de discusiones, se destaca la influencia de la edad en el rendimiento académico. De manera contraria a lo que se esperaba, se encontraron diferencias significativas en cuanto al rendimiento en todas las materias (Lengua Castellana, Matemáticas, Ciencias Sociales y Naturales, Inglés y Religión/Ciudadanía), así como también en la media. Los resultados más altos son para el alumnado entre 16 y 17 años, dándose diferencias con el resto de grupos de edad. Por esta razón, se vuelve a mencionar el trabajo de Pulido y Herrera (2016) como ejemplo en el que se pueden ver diferencias considerando esta variable.

En rendimiento, no se encontraron diferencias significativas entre ambos géneros, ni desde el punto de vista de la media, ni en función de cada una de las materias (Lengua Castellana, Matemáticas, Ciencias Naturales y Sociales, Inglés ni Religión).

Con respecto al rendimiento en función de la cultura/religión del individuo, tal y como se pensaba, se comprueban diferencias estadísticamente significativas en cuanto al rendimiento (media), así como en todas y cada una de las materias, por lo que se podría decir que es un factor influyente en esta variable. En todos los casos, el grupo de alumnos pertenecientes a la cultura/religión cristiana-occidental obtienen mejores resultados.

Así pues, la cultura es un factor a tener en cuenta en el rendimiento en estas asignaturas. Esta realidad ya fue descrita en trabajos anteriores (Pulido \& Herrera, 2016). En este 
sentido, se mencionan las dificultades para la integración socio educativa (Cervini, Dari, \& Quiroz, 2014) y las diferencias de estatus socioeconómico y cultural, las importantes diferencias en cuanto a la lengua materna entre musulmanes y cristianos (Pulido \& Herrera, 2016) del mismo modo que ocurre en otros entornos.

Con respecto al rendimiento en función del estatus, tal y como se pensaba, se encontraron diferencias significativas entre los diferentes grupos. Esto se cumple para la media de rendimiento y para cada una de las materias. En todos los casos, las calificaciones aumentan a medida que lo hace el estatus socio-económico y cultural. Esta realidad se encontró también en experiencias previas (Pulido \& Herrera, 2016). Como justificante se puede volver a mencionar lo comentado en el trabajo de Cervini et al. (2014), donde se destacan las dificultades para la integración socioeducativa y las diferencias de estatus socioeconómico y cultural. También, destacan las importantes diferencias en cuanto a la lengua materna entre musulmanes y cristianos (Pulido \& Herrera, 2016).

Se pone fin a este apartado de discusiones relacionadas con la influencia de la ansiedad sobre el rendimiento académico. De manera contraria a lo que se esperaba, no se encontraron diferencias significativas en cuanto al rendimiento en ninguna de las materias (Lengua Castellana, Ciencias Sociales y Naturales, Inglés y Religión). La única excepción es la materia de Matemáticas, siendo inversamente proporcionales a los niveles de ansiedad. Este trabajo no coincide con otros en los que sí se contempla una relación directa entre los niveles de ansiedad y el desarrollo educativo, influyendo, de forma negativa en su rendimiento académico (Contreras et al., 2005).

Finalmente, en referencia a las limitaciones encontradas en el trabajo, se puede comenzar teniendo en cuenta el contexto en el que este se realizó, pues se establece, de forma clara, la diversidad existente entre dos grupos de culturas distintas. Con la intención de conseguir una superior generalización de los resultados, se ha de considerar de manera más detallada la pluriculturalidad (elementos relacionados con los desiguales patrones de crianza en cada uno de los dos grupos culturales mayoritarios, las costumbres, el modo de vivir la religión, etc.).

El empleo exclusivo de metodología cuantitativa también se debe considerar, dado que este estudio se presta también a métodos cualitativos y mixtos, de nuevo en relación con la multiculturalidad. Por otro lado, de dichas limitaciones se puede desprender la prospectiva de investigación; sería el caso de aquella investigación que considerara introducir otras variables relacionadas con la cultura, la réplica de este trabajo en un contexto diferente y enfocada no solo en el alumnado (profesorado, familias). Finalmente, también se plantea la posibilidad de confeccionar un programa que se pudiera aplicar para la prevención de la ansiedad y para el trabajo de las emociones.

\section{Referencias}

Balongo, E., \& Mérida, R. (2016). El clima de aula en los proyectos de trabajo. Perfiles Educativos, 38(152), 146-162.

Caballero-Dominguez, C. C., Suárez-Colorado, Y. P., \& Bruges-Carbono, H. D. (2015). Characteristics of Emotional Intelligence in a Group of Higher 
Education Students with and without Suicidal Ideation. Revista CES Psicología, 8(2), 138-155.

Cazalla-Luna, N., \& Molero, D. (2014). Inteligencia emocional percibida, ansiedad y afectos en estudiantes universitarios. Revista Española de Orientación y Psicopedagogia, 25(3), 56-73. doi: 10.5944/reop.vol.25.num.3.2014.13858

Cervini, R., Dari, N., \& Quiroz, S. (2014). Estructura familiar y Rendimiento Académico en países de América Latina. Los datos del Segundo Estudio Regional Comparativo y Explicativo. Revista Mexicana de Investigación Educativa, 19(61), 569-597.

Contreras, F., Espinosa, J. C., Esguerra, G., Haikal, A., \& Rodríguez, A. (2005). Autoeficacia, ansiedad y rendimiento académico en adolescentes. Perspectivas en psicología 1(2), 183 - 194

Gómez-Castro, J. L. (1986). Rendimiento escolar y valores interpersonales: Análisis de resultados en E.G.B. con el cuestionario SIV de Leonardo V. Gordon. Bordón, 262, 257-275.

Gómez-Garibello, C. \& Chaux, E. (2014). Agresión relacional en preescolar: variables cognoscitivas y emocionales asociadas. Universitas Psychologica, 13(2), 565-574. doi: 10.11144/Javeriana.UPSY13-2.arpv

Gómez-Ortiz, O., Casas, C., \& Ortega-Ruiz, R. (2016). Ansiedad social en la adolescencia: factores psicoevolutivos y de contexto familiar. Behavioral Psychology/Psicología Conductual, 24(1), 29-49.

Herrera, F. (2000). La inmigración extranjera no comunitaria y la convivencia en contextos concretos: el caso de Ceuta. En Instituto de Estudios Ceutíes, Monografía de los cursos de Verano de la Universidad de Granada en Ceuta (12 ${ }^{\mathrm{a}} \mathrm{ed}$., pp. 357-359). Ceuta: Instituto de Estudios Ceutíes-Universidad de Granada.

Kushnir, J., Gothelf, D., \& Sadeh, A. (2014). Nighttime fears of preschool children: A potential disposition marker for anxiety? Comprehensive Psychiatry, 55(2), 336-341. doi: 10.1016/j.comppsych.2013.08.019

Martínez-Monteagudo, M. C., Inglés, C., \& García-Fernández, J. M. (2013). Evaluación de la ansiedad escolar: revisión de cuestionarios, inventarios y escalas. Psicología Educativa,19, 27-36. doi: 10.5093/ed2013a5

Matesanz, A. (2006). Datos para la adaptación castellana de la Escala de Temores (FSS). Análisis y Modificación de Conducta, 32(144), 521-551.

Moreno-Rosset, C., Arnal-Remón, B., Antequera-Jurado, R., \& Ramírez-Uclés, I. (2016). Anxiety and psychological wellbeing in couples in transition to parenthood. Clínica y Salud, 27, 29-35. doi: 10.1016/j.clysa.2016.01.004

Pinto, B. M. D. C., Dutra, N. B., Filgueiras, A., Juruena, M. F. P., \& Stingel, A. M. (2013). Diferenças de gênero entre universitários no reconhecimento de expressões faciais emocionais. Avances en Psicología Latinoamericana, 31(1), 200-222. 
Pulido, F., \& Herrera, F. (2016). Miedo y rendimiento académico en el contexto pluricultural de Ceuta. Revista de Investigación Educativa, 34(1), 185-203. doi: 10.6018/rie.34.1.207221

Quiceno, J. M., \& Vinaccia, S. (2015). Calidad de vida, fortalezas personales, depresión y estrés en adolescentes según sexo y estrato. International Journal of Psychology and Psychological Therapy, 14(2), 155-170.

Ranoyen, I., Jozefiak, T., Wallander, J., Lydersen, S., \& Indredavik, M. S. (2014). Self-reported social anxiety symptoms and correlates in a clinical (CAP) and a community (Young-HUNT) adolescent sample. Social Psychiatry and Psychiatric Epidemiology, 49, 1937-1949.

Roth, G., Benita, M. Amrani, C., Shachar, B. Asoulin, H., Moed, A... \& KanatMaymon, Y. (2014). Integration of negative emotional experience versus suppression: Addressing the question of adaptive functioning. Emotion, 14(5), 908-919.

Soriano, E. \& González, A. J. (2013). Las competencias emocionales en el ámbito afectivo-sexual de los adolescentes autóctonos e inmigrantes. Revista de Investigación Educativa, 31(1), 133-149. doi: 10.6018/rie.31.1.150531

Spielberger, C. D. (2001). STAIC. Cuestionario de Ansiedad Estado/Rasgo en niños. Madrid: TEA.

SPSS Inc. Released (2011). IBM SPSS Statistics for Windows, Version 20.0. Armonk, NY: IBM Corp.

Victor, M. \& Ropper, A. (2002). Principios de neurología. México: McGraw-Hill. 